\title{
TV/Series
}

12| 2017

Littérature et séries télévisées/Literature and TV series

\section{Quand une série télévisée interroge et joue avec les textes sacrés... Ainsi soient-ils au prisme de l'intertextualité et de la métatextualité}

\section{Benoît Verdier}

\section{OpenEdition}

Journals

Édition électronique

URL : http://journals.openedition.org/tvseries/2142

DOI : $10.4000 /$ tvseries. 2142

ISSN : 2266-0909

Éditeur

GRIC - Groupe de recherche Identités et Cultures

Référence électronique

Benoît Verdier, «Quand une série télévisée interroge et joue avec les textes sacrés... Ainsi soient-ils au prisme de l'intertextualité et de la métatextualité », TV/Series [En ligne], 12 | 2017, mis en ligne le 20 septembre 2017, consulté le 05 mai 2019. URL : http://journals.openedition.org/tvseries/2142 ; DOI 10.4000/tvseries. 2142

Ce document a été généré automatiquement le 5 mai 2019.

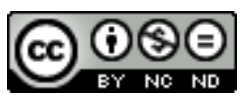

$T V /$ Series est mis à disposition selon les termes de la licence Creative Commons Attribution - Pas d'Utilisation Commerciale - Pas de Modification 4.0 International. 


\title{
Quand une série télévisée interroge et joue avec les textes sacrés... Ainsi soient-ils au prisme de l'intertextualité et de la métatextualité
}

\author{
Benoît Verdier
}

1 Second semestre 2012, alors que la controverse sur le mariage pour tous commence à se structurer (Cervulle, 2013), une chaîne hertzienne porte le religieux à une heure de large audience dans un format et un genre particulier. Si le support interroge peu au regard de son succès ces dernières années ${ }^{1}$, il en va différemment du sujet central au regard de l'hégémonie des fictions sérielles investissant plus massivement les métiers de la sécurité, de la justice et de la santé. Investir le religieux dans un produit culturel de masse était un pari audacieux pour la production et le diffuseur. D'ailleurs, le titre - Ainsi soient-ils (Arte, 2012-15) - traduit cette impétuosité auctoriale jouant avec le texte sacré : un pastiche au pluriel de la traduction française du mot «Amen ». La racine sémitique à l'origine de ce mot exprime la Foi, la confiance en Dieu. Par cette expression, les fidèles se placent sous cette protection divine dans une adhésion collective et individuelle à un dogme. En outre, ce titre fait état d'une interjection chrétienne - parodiée - qui paradoxalement marque la fin (d'une prière). La fiction sérielle, dès son titre, propose déjà des déplacements conséquents de sens. Plus qu'un dérivatif parodique, il s'inscrit dans une proposition critique, reconfigurant ainsi les frontières avec les textes pétrifiés dans leur tradition (dogmatique).

2 L'objectif de cet article est d'interroger la manière dont une série télévisée s'empare des textes sacrés. Autrement dit, il s'agit d'investiguer comment un produit culturel de masse ayant pour objet le croire, la formation de ses ministères etc, investit la littérature liturgique et théologique en questionnant la place, la représentation et les fonctions qu'elle lui donne. Partant, nous en retiendrons une définition relativement large. Les 
écrits de la parole divine (celle rapportée à Moïse), communément appelés l'Ancien Testament, sont associés aux écrits réalisés a posteriori -les évangiles - et rédigés par les apôtres rapportant leurs souvenirs en mélangeant allègrement le temps, les lieux et parfois les événements. Aussi, dans notre acception, l'Ancien Testament côtoie et se mêle avec le nouveau Testament, sans distinction hiérarchique: cet ensemble est généralement dénommé la «Bible chrétienne ». À ces textes relevant du dogme, nous ajoutons les ouvrages rédigés par, ou consacrés à, certaines figures canoniques de la chrétienté (ceux de Saint Augustin par exemple), mais également le livre des jours - lectionnaire contenant les passages des textes religieux à lire pour chaque office chaque jour de l'année -. Si cette définition étendue de la littérature sacrée peut représenter un biais à notre analyse, la série, néanmoins, n'en propose ni une distinction claire, ni une classification hiérarchisée. Aussi, nous l'assumons pour examiner le texte sacré non pas dans ses spécificités intrinsèques ou exégétiques, ni dans leur ordonnancement pendant les rituels, mais plutôt dans l'utilisation et la représentation proposée par cette série et, par conséquent, les questionnements critiques qu'elle suscite.

3 La série ${ }^{2}$ suit l'arrivée, la vie et la transformation de cinq jeunes hommes en première année dans un séminaire de l'Église catholique en plein cœur de Paris dans le quartier latin. Issus de milieux sociaux différents, ayant des parcours et motivations très diverses, ce sont cinq adultes bien ancrés dans leur époque (contexte du XxI e siècle en France) que tout oppose mais que la Foi rapproche. Le dossier de presse avec les interviews des scénaristes et/ou du producteur, annonce clairement que cette fiction ne s'adresse pas uniquement à une audience catholique (pratiquante ou non). Le public attendu se veut le plus large possible, dépassant les confessions, croyant ou non. Nous trouvons dans cette promesse de la série le caractère syncrétique des produits culturels télévisuels. En outre, le choix de cette institution religieuse n'est pas si anodin. En effet, depuis de nombreuses années, l'Église catholique est sous le feu des médias en raison de toutes les affaires qui la concernent: pédophilie, mariage des prêtres, secret du Vatican, Banque du Vatican... Selon les auteurs, il ne s'agit pas de faire un pamphlet pour/ou contre l'église catholique, mais bien de « créer des personnages et les ancrer dans la réalité, dans le monde, dans la société contemporaine $»^{3}$ pour traiter de l'engagement au sens large (dans une communauté, pour une opinion...).

4 Le corpus étudié se compose des 16 épisodes de 52 minutes des deux premières saisons. Notre travail s'inscrit notamment dans la lignée de ceux de Sabine Chalvon-Demersay (notamment 1999, 2000, 2011) qui sondent les fictions pour investiguer le social. Aussi, nous avons privilégié une analyse au croisement de l'analyse sémio-narrative et de contenu (Jost, 2007, Esquenazzi, 2010) qui s'appuie également sur les théories développées par G. Genette $(1972,1982)$.

5 Nous procédons en trois temps. Premièrement, les modalités de monstration de cette littérature sacrée permettent de repérer son empreinte fondamentale dans l'univers professionnel mais également dans la narration. Puis, nous abordons la fonction de métatextualité à travers le dispositif homilétique, utilisé dans cette série comme procédé narratif. Enfin, les fonctions d'intertextualité et d'interdiscursivité illustrent un dialogue interreligieux et un croire contemporain bricolé. Ces différentes transactions qui se font jour dans la série sont analysées pour révéler la puissance créatrice et subversive de ce genre télévisuel. Il s'agit de démontrer sa capacité à interroger le canon considéré non pas comme une forme d'étrangeté érigée au panthéon de la tradition mais bien doté d'une 
certaine sagacité à proposer des anagogies contemporaines à visée introspective et réflexive, voire de susciter un questionnement critique social.

\section{Modalités de monstration des textes sacrés}

\section{Un marqueur du temps}

6 Le pilote propose la rentrée des jeunes séminaristes. Il permet la présentation de l'ensemble choral des personnages principaux et l'arène des arcs narratifs, le séminaire des Capucins. Les deux premières saisons s'étalent sur deux années universitaires classiques, marquant ainsi deux années de formation de septembre à juin. Les saisons mais également les diverses fêtes qui ponctuent l'année civile se manifestent dans la série : moments particulièrement liés à notre culture judéo-chrétienne (de la toussaint à Pâques en passant par Noël). Ce procédé classique de narration fictionnelle sérielle permet d'égrener le temps en parallèle des événements qui rythment la vie des téléspectateurs en développant ainsi le caractère réaliste et authentifiant (Esquenazi, 2010, Jost, 2007) des fictions.

7 La temporalité est scrupuleusement suivie : au delà du calendrier social, la série suit également le calendrier liturgique. Les scénaristes respectent fidèlement les textes proposés par le Livre des Jours. A l'écran, ce livre apparaît deux fois en tant qu'objet. Si ce détail ne peut être décodé que par les initiés, il participe du réalisme. Particularité de la série inhérente à son thème central, le Texte est utilisé et fonctionne comme un marqueur de temps.

\section{Usage exclusif des textes sacrés}

8 Cette littérature canonique, dans la série, reste strictement réservée aux initiés d'une part de genre masculin et de l'univers ecclésial d'autre part. En effet, les Textes sacrés seraient le privilège des hommes. Ces derniers apparaissent comme les seuls à avoir accès aux textes sacrés (et d'ailleurs aux livres en général). Ils les étudient et sont détenteurs du savoir livresque et particulièrement ici du dogme. Dans cette fiction sérielle, la littérature sacrée est donc associée au pouvoir masculin et seuls les membres de l'Institution ecclésiale, particulièrement les séminaristes, les pères en font un usage quotidien : ils l'ont en main, la rangent, la lisent, s'en inspirent (fig. 1 et 2). À cet égard, la bibliothèque du séminaire est un lieu récurrent. De plus, pour prendre un exemple parmi tant d'autres, dans les plans successifs du dernier épisode de la première saison (1.8), parmi l'ensemble des personnages présents, seuls les représentants de la religion catholique romaine disposent de cette littérature : monseigneur Roman, notamment, installé à son bureau, est concentré sur la lecture d'une édition ancienne. 
Fig. 1. Valise du père Bosco pour son voyage au Vatican (1.4)

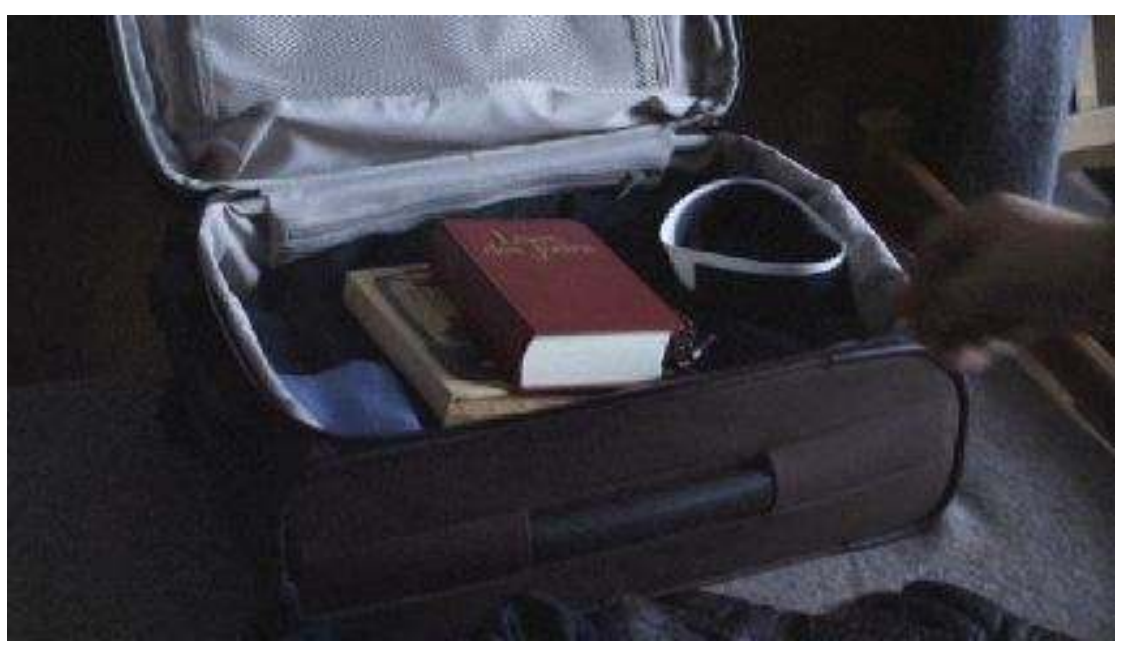

Fig. 2. Père Valéry ramène les livres qu'il emprunte secrètement à la bibliothèque (1.5)

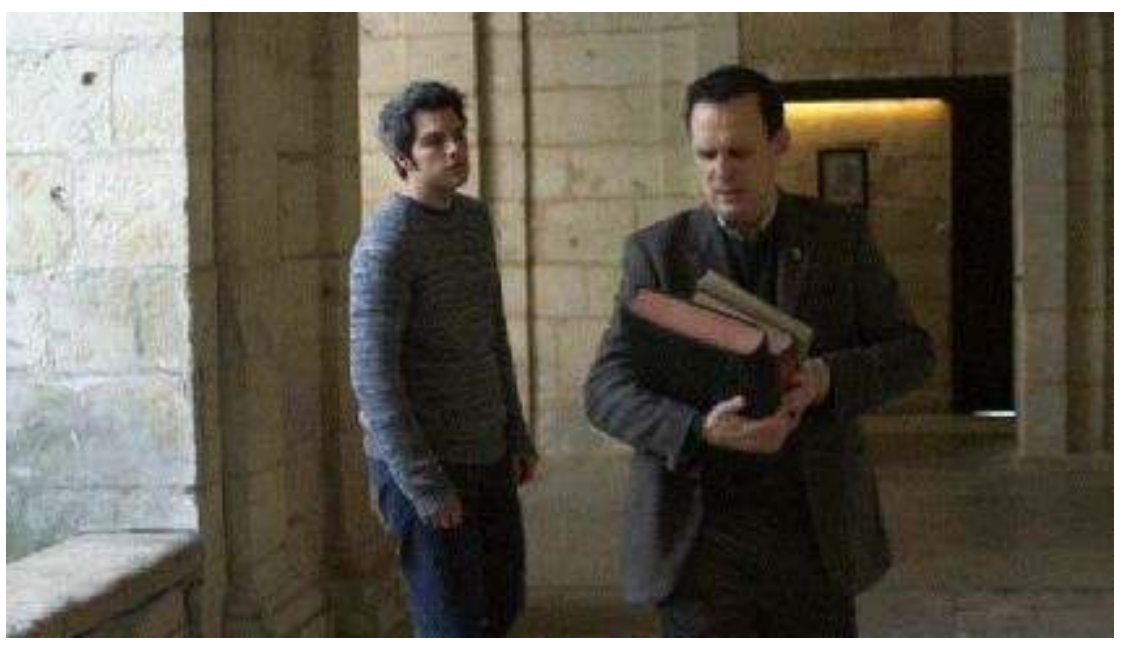

9 Néanmoins, une seule exception demeure: le père de Raphaël, un des cinq jeunes séminaristes, est le seul élément de la société civile qui a l'objet livre en main. Suite au décès de son fils ainé, il se réfugie dans la religion (1.7). Assis dans un fauteuil, il lit la Bible et récite un psaume à son second fils. Ce n'est pas anodin. Raphaël et un autre jeune séminariste se situent dans cette figure singulière que Danielle Hervieu-Léger (1999) nomme du paroissien - des individus à qui la famille a transmis en « héritage » cette Foi et qui s'inscrivent territorialement dans une paroisse, une communauté, une culture -. Issue d'une lignée bourgeoise (voire aristocrate), sa famille appartient «au grand capital » comme nous l'indique Raphaël (1.5). La famille loge dans un château en proche région parisienne, détient une société d'investissement, une holding au nom de la famille dont le siège social semble se situer à la Défense. Une des intrigues se développe autour de ce monde des affaires, de l'argent en lien avec la justice, l'état et la politique (inscription de la série dans l'actualité). En outre, Raphaël n'est pas une exception au sein de sa famille puisqu'un aïeul a eu une fonction ecclésiastique. Dans ce cas, la religion est reçue, acceptée et transmise de génération en génération (dans le sens des Héritiers de Bourdieu, 1964). 


\section{De la nécessité d'une socialisation}

10 Mise à part la famille Chanseaulme, l'ensemble des personnages issus de la société civile semble considérer négativement cette littérature. José, un des cinq jeunes séminaristes, intègre le séminaire après un séjour en prison où il s'est converti au catholicisme (1.1). C'est dans ce lieu qu'il s'est socialisé avec les écrits, le dogme, etc. Il aurait reçu l'appel de Dieu en se réfugiant dans une église après le meurtre d'un homme. Alors qu'il discute avec un de ses amis, une sorte de petit délinquant, celui-ci vitupère : « ils doivent le dire, là, dans ta bible ». La littérature sacrée est ainsi objectivée et surtout mise à distance : un objet lointain, inconnu et sans attirance.

11 D'ailleurs, en termes de socialisation, José, au regard de son parcours, n'a pas les codes : il invective le père Fromenger (Supérieur du séminaire) qu'il a suivi dans la rue afin de lui donner sa candidature : « Tolle, lege ; prends et lis ; c'est de ça dont parle Saint Augustin làdedans ». Cette locution adverbiale « là-dedans » désigne vulgairement le livre tenu par le père Fromenger, celui des mémoires de Saint Augustin. Le plan précédent montrait l'hésitation du père entre deux éditions de cet auteur. A la fin de leur dialogue, le père supérieur lui offre le second livre sans désigner verbalement l'objet. C'est la seule fois où le texte sacré est mis à distance par les hommes d'église. Précisons qu'à ce moment de la série, José n'est pas encore séminariste, un détail qui a son importance tant les clercs ont une réelle affinité avec l'objet.

Cette socialisation passe également par un usage très codifié à travers une référenciation normalisée telle que verset $\mathrm{X}$ selon $\mathrm{Y}$, ou bien évangile selon $\mathrm{X}$. En outre, la déférence envers le manuscrit, "ce verbe fait chair en la personne de Jésus Christ », " la Parole », atteste l'autorité du dogme et consacre l'idée commune d'une religion du livre. L'objet n'est pas qu'un livre ; il représente plus que le signifiant qu'il compile. La relation qui lie le clerc à l'objet est singulière. De tous les moments du quotidien et du rituel, il est au cœur de la relation au métier. C'est le terreau nourricier de leur être professionnel et personnel. L'objet n'en est pas un, il représente beaucoup plus : il est la révélation de Dieu, « le verbe fait chair ».

\section{Discours homilétique : métatextualité professionnelle}

Il s'opère une double présentification ${ }^{4}$ du texte sacré à la fois par l'objet et par la pensée qu'il contient, le texte lui-même sous forme d'extrait. Un père du séminaire précise, alors qu'il s'apprête à ranger la bibliothèque (1.5) : «Je n'ai absolument pas le droit de sortir ces ouvrages de la bibliothèque, mais pourtant c'est plus fort que moi, j'en emporte tous les soirs dans ma chambre ». À la fin du dernier épisode de la saison 1, la séquence propose la dernière homélie du père Bosco. Le montage alterne le séminaire et la société extérieure (notamment la famille des différents séminaristes). Le manuscrit, en tant que signifiant pour caractériser l'objet et signifié en termes de Parole divine, sera utilisé pour représenter la Foi et le métier. Emmanuel, un des cinq séminaristes qui va quitter ce lieu de formation pour revenir à la vie civile en tant qu'enseignant-chercheur en archéologie, range sa bible dans son bureau d'enfant, dans sa chambre d'enfant (1.8). Sa vocation (et ce métier en devenir) est dorénavant dans un coin de sa tête : cette expérience de Foi a construit l'homme qu'il est (fig. 3). Figure classique du cinéma pour signifier en une 
image, la métonymie du métier s'est réalisée sur le texte. Le choix ne s'est donc pas porté ni sur l'habit, ni sur une croix mais sur la Bible, synecdoque fusionnant le dogme et la profession.

Fig. 3. Emmanuel range sa bible dans son bureau (1.8)

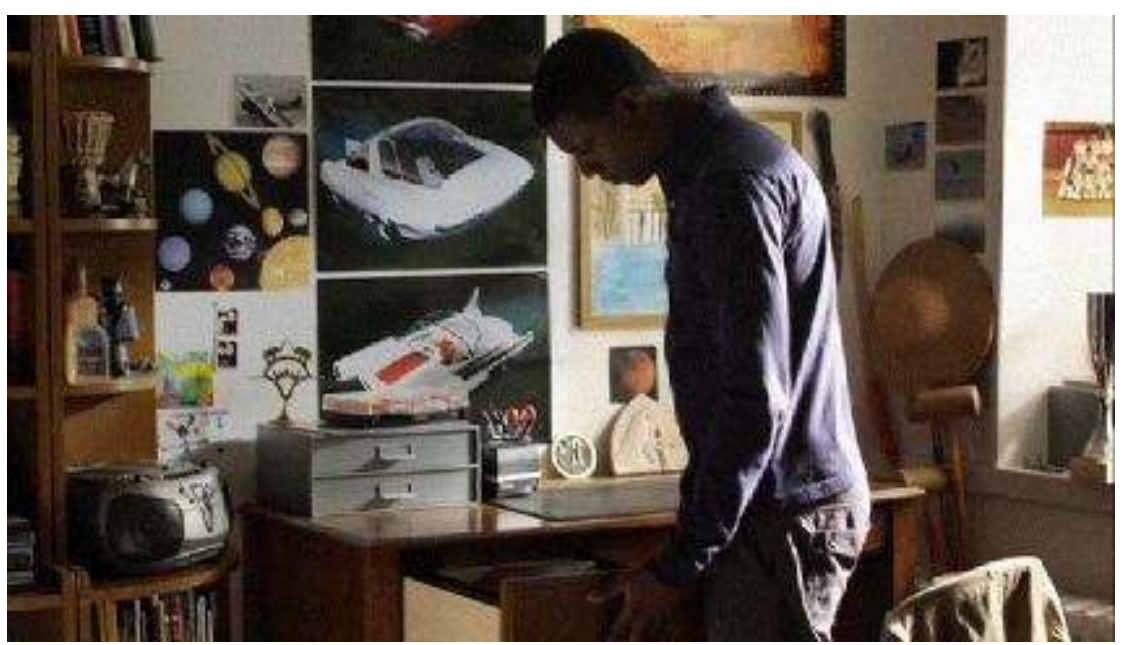

Lieu de formation, le séminaire enseigne l'exégèse des textes. Les cours sont l'occasion d'en développer une connaissance fine, d'en approfondir la signification et la portée dans les actes de la vie quotidienne de leurs futurs administrés. Les hommes d'église se réfèrent souvent aux textes par le biais d'une citation pour éclairer (ou se positionner dans) une situation de communication. Dans la vie de tous les jours comme lors de rituels (messe), il s'agit pour le clerc d'exemplifier ses propos par le biais d'une phrase dont la valeur est alors augmentée.

Inhérente à cet univers professionnel où le verbe est omniprésent et omnipotent, cette transaction particulière fait partie intégrante de la professionnalité de ces fonctionnaires du culte. À chaque épisode, un temps homilétique d'une durée fluctuante ${ }^{5}$ (deux minutes en moyenne) est proposé et réalisé par les pères Bosco, Fromenger ou les séminaristes. Un concours entre les séminaristes est même organisé (2.5). L'homélie sert de support à la narration (Verdier, 2016) : la référence au Texte clôt à chaque fois la (ou les) intrigue(s) externe(s) et/ou interne(s). Elle concentre en mots et met en perspective les événements passés dans un montage particulier favorisant les personnages (à travers des plans américains ou des portraits) au détriment de la littérature sacrée placée sur l'ambon. Le Texte en tant qu'objet se fond alors dans le décor sans aucune considération. Dans ce cadre, c'est la matérialisation d'une pensée par le biais de la citation et la plus-value de l'hypertexte proposé qui importe.

D'une part, par l'ouverture symbolique du livre - en tant qu'objet sur le pupitre ou comme une citation en arrachant un passage à l'hypotexte -, cette parole homilétique se trouve alors légitimée. D'autre part, avec le commentaire qu'il apporte à cet extrait, le clerc recrée un nouveau texte, comme un métadiscours sur le discours. La série s'appuie largement sur cette

relation, on dit plus couramment de " commentaire ", qui unit un texte à un autre dont il parle, sans nécessairement le citer (le convoquer), voire, à la limite, sans le nommer [...] C'est, par excellence, la relation critique » (Genette, 1982, p. 10). 
Indissociable de la pratique religieuse, cette fonction de métatextualité est particulièrement visible et lisible lors des offices religieux, avec un agencement et un ordonnancement des textes bien précis. En outre, dans cette série, ce discours s'organise également à partir d'un autre texte (profane), celui de l'expérience vécue du (ou des) personnage(s).

Pour paraphraser Genette, ce métadiscours apparaît comme une série d'« interventions, directes ou indirectes, du narrateur à l'égard de l'histoire» qui peut prendre la configuration "d'un commentaire autorisé de l'action: ici s'affirme ce qu'on pourrait appeler la fonction idéologique du narrateur» (1972:p. 262-263). Partant, cette transaction proposée par la série nous permet de mettre en exergue deux pôles d'action de ces nouveaux textes : introspectif pour les personnages (narrataires intradiégétiques) et réflexif pour les téléspectateurs (narrataires extradiégétiques). En effet, d'une part, le discours homilétique reconsidère les éléments du passé (proche et/ou lointain) digérés et rétrocédés à la lumière du Texte sacré en tant que métadiscours. Ces moments homilétiques fixent le caractère évolutif des personnages et prennent alors la même valeur que peuvent avoir le flashback (ou analepse dans la terminologie structuraliste proposée par Genette) ou le flashforward (ou prolepse) sur la construction et la complexité du personnage évolutif (Verdier, 2016). Et, d'autre part, le sermon est un discours circonstancié proposant aux fidèles ce qu'il faut croire et faire pour la gloire de Dieu et pour le salut des Hommes. Dans la série, l'homélie relève plus d'une prédication d'ordre général, à portée universelle. Constitués d'éléments discursifs atemporels, ce sermon favorise l'individualisation dans un nous collectif (Verdier, 2016). Ce discours homilétique s'oppose donc au discours catéchétique qui propose, comme le dit Lemieux :

un approfondissement de la Foi et surtout, sa concrétisation dans l'organisation de la vie chrétienne, [et] suppose une communauté de la Foi (1987, p. 20).

19 Ici, cette parole kérygmatique se veut universelle, et dans notre cadre, intégrative. Elle impose un rythme plus lent, lié d'une part au rituel, mais surtout plus propice à la réflexion. Il s'agit de s'adresser à un auditoire très large, pas forcément catholique, dont la religiosité héritée, choisie ou bricolée peut être différente. Cette fonction de l'utilisation des textes sacrés proposée par la série permet au spectateur d'appréhender, de donner du sens à ce qu'il a vu (ce qu'il a pu vivre par procuration avec les personnages et comment cela résonne en lui) dans un moment comme suspendu dans le temps (Verdier, 2016).

\section{Du dialogue inter-religieux à l'éloge du bricolage}

\section{Moult transactions intertextuelles}

S'il n'y a pas de scribes comme dans le film Le nom de la rose (1986), adaptation du roman d'Umberto Eco, la référenciation au Texte en tant que signe est pourtant présente et apparaît sous une forme inattendue. En effet, le Père Fromenger, père supérieur du séminaire, s'adonne (dans trois épisodes) à la calligraphie chinoise (fig. 4). L'art de l'écriture («Les Saintes Écritures» copie manuscrite de la parole Divine) se rapporte à d'autres confessions aussi bien en Orient, en Asie ou en Europe. Son souhait, son rêve, est de traduire en calligraphie chinoise le Cantique des Cantiques, défi de taille en ligne de fuite de ce personnage. Constitué d'une suite de poèmes, de chants d'amours entre une femme et un homme, ce livre, de la Bible est à la fois poétique et explicite. Certains y 
voient l'amour physique entre un homme et une femme, d'autres, considérant l'attirance des corps méprisable, la relation qu'entretient le Christ avec l'Église ou avec l'âme humaine. La série, avec le père Fromenger, penche pour la première alternative : «J'ai trouvé dans un poème de Li Bai la même image que celle du Cantique des Cantiques "tes lèvres ressemblent à un fil écarlate” » (1.3) ou bien lorsqu'il évoque sa traduction : «les multiples formes que prend l'amour entre un homme, une femme et le cosmos me posent d'insurmontables problèmes de vocabulaire » (2.4).

Fig. 4. Père Fromenger et la calligraphie chinoise (1.3)

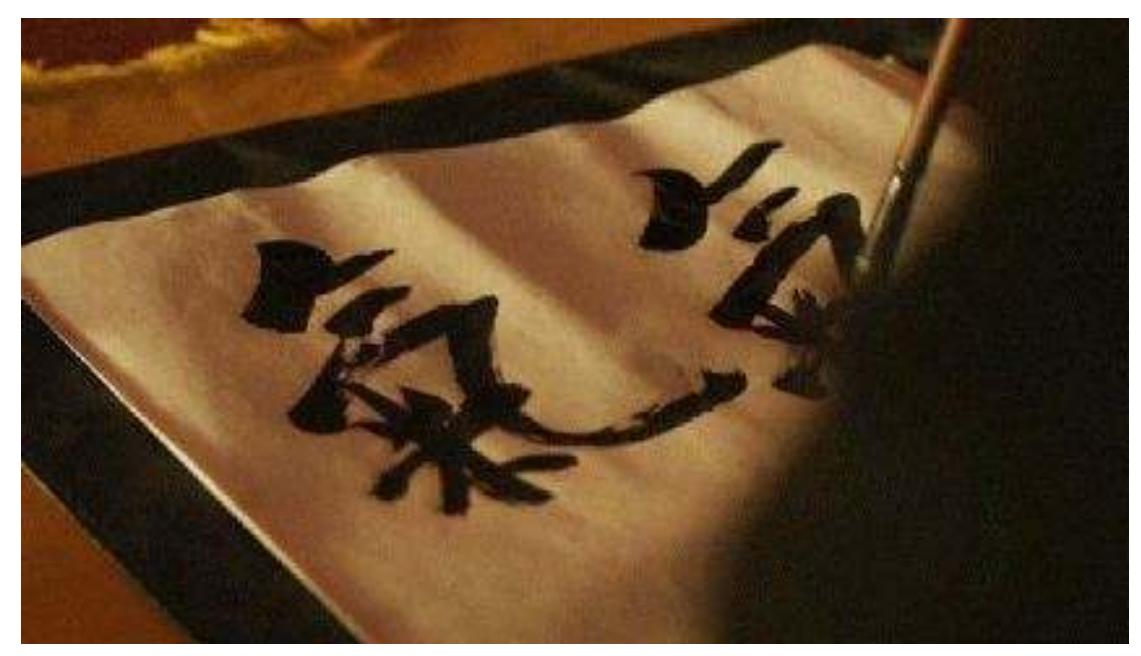

21 Une autre transaction, sous forme de brouillage entre le signifiant et le signifié, fait la part belle à la tradition juive. Les nombreuses références talmudiques sont liées à un personnage secondaire, un patient ayant sympathisé avec José, l'un des cinq séminaristes, dans un hôpital de convalescence. Bien qu'élie soit non pratiquant, il l'emmène à la Sim'hat Torah (2.3). Cette fête spécifique de la religion juive qui célèbre les textes sacrés est filmée comme un défilé festif et joyeux où les hommes chantent et dansent avec les rouleaux de la Torah (fig. 5). Dans ce cadre, le texte sacré est porté aux nues dans un moment d'allégresse collectif genré (puisqu'exclusivement masculin). C'est alors le signifié de l'objet livre dans sa globalité qui est donné à voir: la Torah est un texte manuscrit. Il s'opère une sorte de vénération du texte avant de passer à sa lecture. La référenciation au texte n'est pas réalisé dans le signifié d'une citation, mais bien par l'objet livre en tant que signifiant. 
Fig. 5. Sim'hat Torah (2.3)

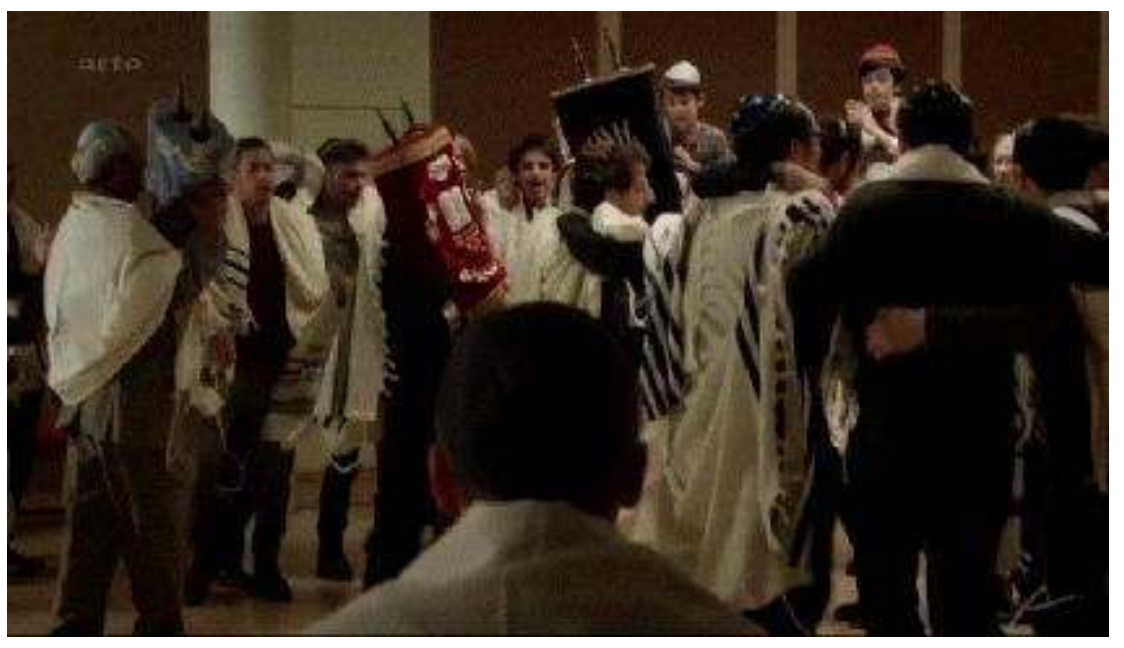

L'action des séminaristes et d'un personnage libertaire auprès d'un groupe de sanspapiers dans un squat est une des intrigues externes de la première saison. Pour étouffer une querelle au sein du groupe de squatteurs, à la manière d'un discours homilétique, José intervient et les interpelle sur le texte relatif à la construction de la tour de Babel (1.6). José, en conclusion, propose une interprétation talmudique et non catholique de cet extrait. S'il est bien commun aux deux traditions monothéistes (puisqu'issu de l'ancien testament), son commentaire diffère sensiblement selon les deux religions. Ce processus d'interdiscursivité donne une autre dynamique aux Textes sacrés, trop souvent figés dans un dogme sans possibilité de discourir ensemble. En effet, la série propose dans un même plan, au même niveau, deux métatextes de deux confessions différentes à partir d'un seul et même hypotexte, l'Ancien Testament, qui leur est commun et dont ils tirent leur origine.

D'autres éléments viennent également "parasiter » cette littérature sacrée, notamment des textes de type profane par exemple d'ordre psychanalytique :

PÈRE CHEMINADE. « De mon temps, nous appelions ça confession mais aujourd'hui, nous appelons ça réconciliation. Et, c'est ça le sens profond de ce que vous allez vivre. Oubliez les vieilles notions de pénitences. La réconciliation est un coup génial souvent imité et jamais égalé. Demandez aux psychanalystes.

RAPHAEL (en messe basse à son collègue). C'est Freud qui nous a tout piqué. (1.3)

S'ajoutent également des symboles païens (voire religieux). En effet, le marketing de la série propose certaines images (affiche, jaquette DVD) montrant un personnage dont on ne voit que le corps revêtu d'une aube blanche, tenant un calice ou une croix dans une main tatouée (fig. 6 et 7). En saison 1, José, un jeune séminariste, se change dans un vestiaire et laisse apparaitre sur son épaule droite des ailes (1.2). Le tatouage relève d'une démarche de construction identitaire et de présentation de soi, comme le souligne David Le Breton (2002) qui l'analyse comme un ajout de sens que l'individu applique sur son corps pour remédier aux pertes de repères engendrées par la modernité.

La marge de création [de l'individu] s'amplifie d'autant plus que la culture ambiante est sans épaisseur réelle et fonctionne à la manière d'un vaste supermarché de biens matériels et symboliques » (Le Breton, p. 16).

Comme nous l'indique sa promesse, cette fiction sérielle ne se veut pas segmentante. Au contraire, avec ces transactions, elle développe une rhétorique d'un croire universel 
(dépassant les clivages confessionnels) «bricolé » (Lévi-Strauss, 1960) face à un monde désenchanté pour reprendre l'expression de Gauchet (1985). D'ailleurs, à travers ce pluralisme fécond, la série médiatise le rapport de la société à elle-même, de l'individu à lui-même (Verdier, 2014).

Fig. 6. Jacquette DVD Saison 1

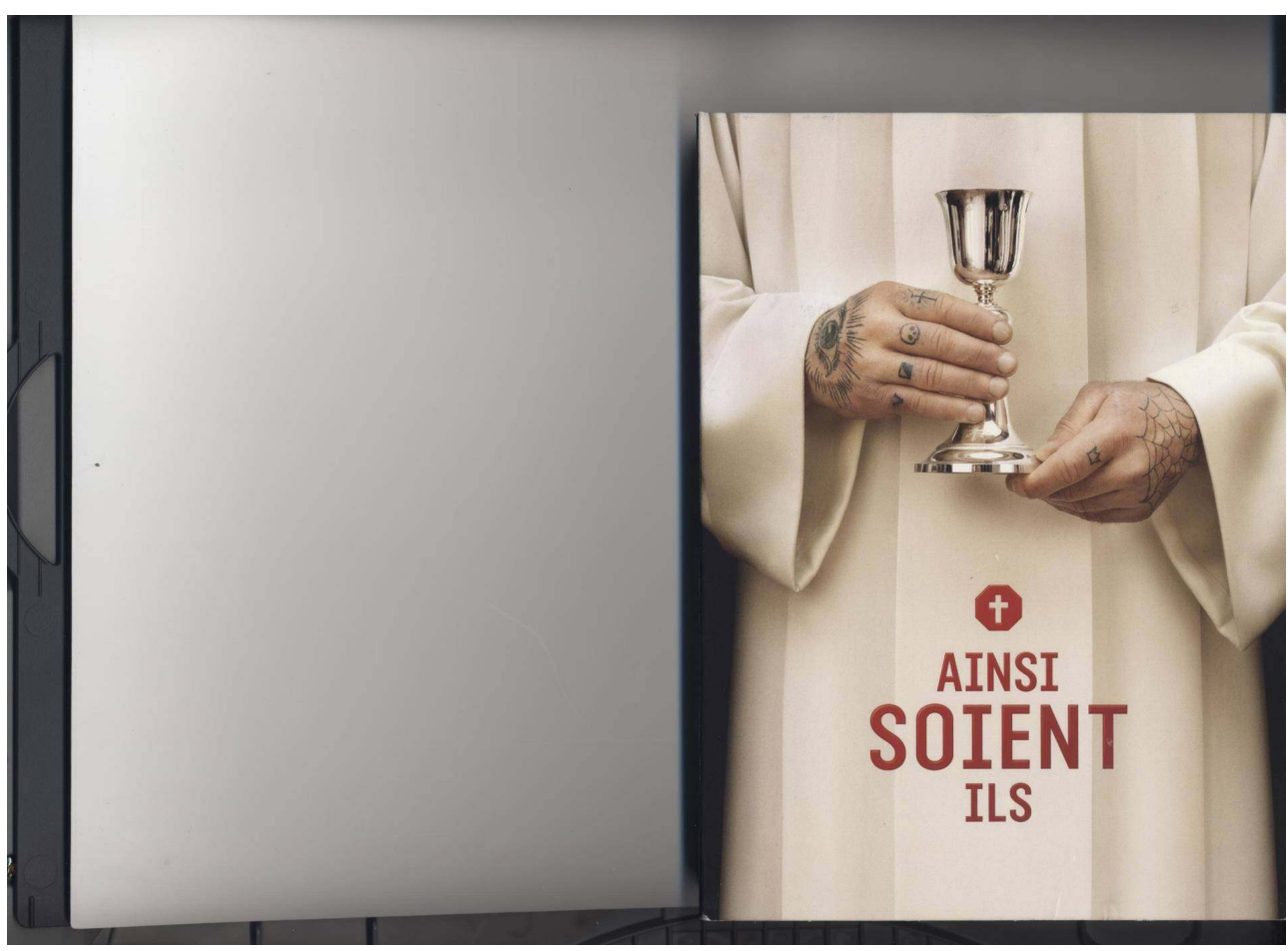

Fig. 7. José dans le vestiaire (1.2)

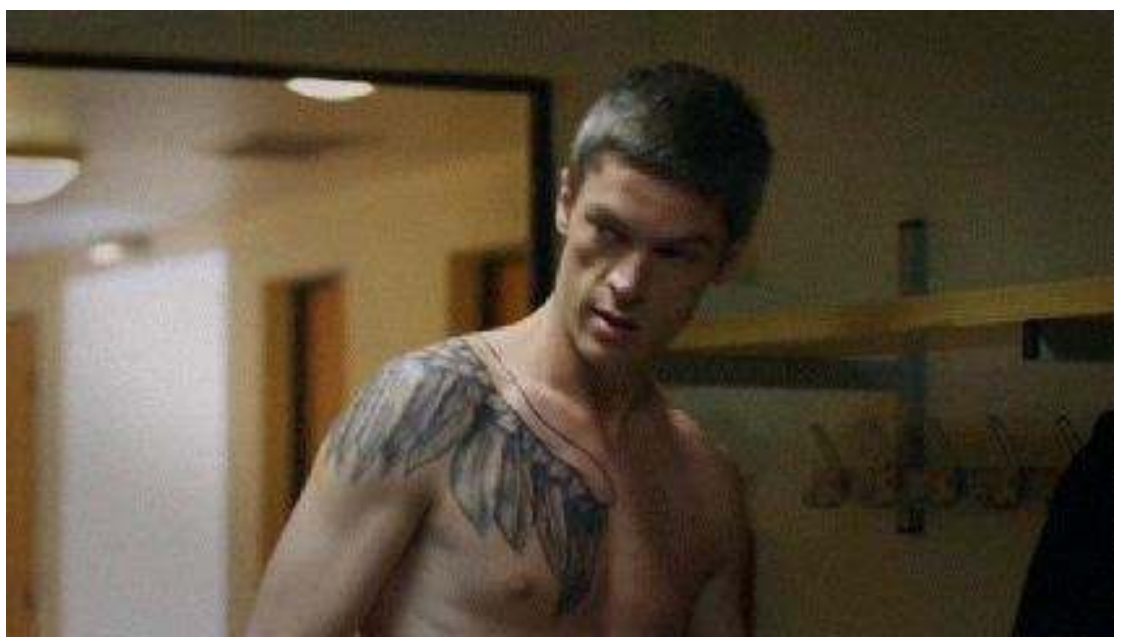

\section{Critique genrée de l'autorité}

Si les textes sacrés sont associés au pouvoir masculin, la série pose pourtant clairement la question de "l'autorité masculine" dans le dernier épisode de la première saison. Raphael Chanseaulme tente de rompre sa relation avec la femme d'un de ses amis, avec 
qui il vient de faire un pacte : il sort définitivement de leur vie et en contrepartie, il évite à sa famille une affaire politico-financière. Lors de cet échange, Raphaël utilise un extrait du Texte, et la jeune femme lui répond:

CLAIRE BODDET. Mais? Tu ne peux pas te servir des Évangiles comme ça! On ne peut

pas servir deux maîtres à la fois : ça fait référence à Dieu et à l'argent. (1.8)

Cette citation ${ }^{6}$ 'est ici qu'un pré-texte - prétexte - pour exprimer un souhait de rupture (plus contraint que choisi). Comme nous l'indique Antoine Compagnon (1979), la citation n'a plus besoin d'auteur pour se légitimer; elle se justifie intrinsèquement de par son propre contenu, comme si elle se suffisait à elle même (1979). Incarnant l'autorité, l'homme utilise la citation pour définitivement asseoir son primat, et son choix se porte sur un extrait d'un texte consacré issu du monde professionnel dans lequel il évolue. En créant une relation entre un hypotexte et un hypertexte, ce procédé, selon Genette (1982), relèverait plutôt de la transtextualité, (ou comme un «cas limite» d'intertertextualité selon Jenny, 1976), voire de l'hypertextualité puisque dans notre exemple, le jeune homme opère un pastiche de la citation choisie, produisant dans la communication en train de se faire un flou sémantique. Cette confusion de sens, qui sert ici la lâcheté de l'homme, va être récupérée par la femme pour prendre l'autorité (d'exégèse). Elle a une connaissance suffisante du Texte pour voir ce qui se trame et ainsi proposer une signification à la phrase, mais aussi et surtout à cette situation de communication. Cet exemple montre la capacité d'une série télévisée à interroger les rapports sociaux en termes de genre dans la sphère privée comme professionnelle, particulièrement au sein d'une institution telle que l'Église catholique romaine française dans laquelle ils font débat.

\section{Conclusion}

Tout au long de ce travail, nous avons montré l'aptitude de cette série télévisée à pervertir des textes sacrés, à en jouer. Tout d'abord, ils servent de support à la narration : leur usage aide au réalisme de l'univers dépeint, marque une temporalité authentifiante, et sert d'idiome figuratif (Gadéa, Cléau, 2013) du métier (voire plus largement d'un engagement spirituel à une Église). À l'instar des divers accessoires positionnés sur la blouse blanche des soignants, la littérature sacrée, comme objet livre mais également comme citation, sert de signifiant incarné pour la représentation de ces professionnels.

La métatextualité fait partie intégrante de leur professionnalité; cette fonction est inhérente à ce monde social. Exégètes, les clercs connaissent finement la littérature sacrée pour se servir d'extraits pertinents en fonction des situations. D'ailleurs, le rituel intègre en partie cette fonction par la présence d'un discours homilétique dont la série va user comme d'un dispositif narratif proposant des moments introspectifs et réflexifs.

L'intertextualité et l'interdiscursivité sont féconds et innovants dans cette série française. En effet, à côté des textes de tradition catholique, d'autres gravitent. Le judaïsme, la calligraphie chinoise mais également des références profanes (Freud ou les tatouages) se manifestent. Tous ces textes (sacrés et profanes) dialoguent entre eux et proposent un ensemble hétéroclite de religiosité qui corrompt le point de vue. Dans cette fiction sérielle, point de discours catéchétique, point de parole exégétique, tous les signifiés drainent large et polluent la référenciation à l'instance religieuse. C'est une vision post- 
moderne du croire, un croire reconstruit, bricolé où se juxtaposent les références religieuses ou philosophiques qu'elle propose (Verdier, 2014).

Le rapport au religieux est très individualisé, c'est un rapport libre de toute appartenance et de relations sociales qui parle à la sphère privée de l'intime. Cette série incarne le positionnement républicain, laïc, historiquement construit, où le croire est considéré comme personnel et privé, et son expression dans l'espace public encadrée. Thème central de la série, l'engagement n'est pas vécu comme une évidence communautaire mais plutôt comme une mise à l'épreuve individuelle.

Ces pratiques intertextuelles dans cette série télévisée rendent donc possible des retours, des critiques sur, mais également entre, des œuvres qu'elles évoquent et, par conséquent, proposent une rencontre providentielle entre des cultures trop souvent séparées en réinventant un dialogue inter-religieux. Cette fiction sérielle regorge d'exemples de ce type et joue donc sur l'intertextualité et l'interdiscursivité en troublant les frontières entre les religions.

Enfin, ces différentes fonctions, éprouvées tout au long de la série, sont éclairantes de la capacité d'une œuvre sérielle à proposer des dialogues inédits entre des œuvres pétrifiées dans leur posture dogmatique ou avec des textes profanes. En effet, seule une transaction hors rituel propose une réflexion critique de la question de la femme dans l'Institution malgré l'omnipotence masculine essentiellement ecclésiale- et par extension dans la société.

\section{BIBLIOGRAPHIE}

CHALVON-DEMERSAY Sabine, « La confusion des conditions. Une enquête sur la série télévisée Urgences ", Réseaux, volume 17, n 95, 1999, p. 235-283.

CHALVON-DEMERSAY Sabine, « Pour une approche sociologique des œuvres de fiction télévisée », in De l'écrit à l'écran : littératures populaires : mutations génériques, mutations médiatiques, éd. Jacques MIGOZZI, Limoges, PULIM, 2000, p. 851-864.

CHALVON-DEMERSAY Sabine, « Enquête sur l'étrange nature du héros de série télévisée », Réseaux, volume $1, \mathrm{n}^{\circ}$ 165, 2011, p. 181-214.

COMPAGNON Antoine, La seconde main, ou le travail de la citation, Paris, Seuil, 1979.

ESQUENAZI Jean-Pierre, Les séries télévisées. L'avenir du cinéma?, Paris, Armand Colin, 2010.

JOST François, Introduction à l'analyse de la télévision, Paris, Ellipses, 2007.

GADÉA Charles, CLÉAU Hélène, « Le drame social du travail à l'hôpital », in Les mondes pluriels de Howard S. BECKER, éd. Marc PERRENOUD, Paris, La Découverte, 2013, p. 49-66.

GENETTE Gérard, Figures III, Paris, Seuil, 1972.

GENETTE Gérard, Palimpsestes, la littérature au second degré, Paris, Seuil, 1982.

HERVIEU-LÉGER Danielle, La religion en mouvement : le pèlerin et le converti, Paris, Flammarion, 1999. 
JENNY Laurent, « La stratégie de la forme », Poétique n² 27, 1976, p. 257-281.

LEMIEUX Raymond, « Charisme, mass media et religion populaire. Le voyage du pape au Canada », Social Compass, volume XXXIV/1, 1987, p. 11-31.

LÉVI-STRAuss Claude, La Pensée sauvage, Paris, Plon, 1960.

VERDIER Benoît, « Le discours homilétique comme dispositif de narration », Télévision, n 7, 2016, p. 101-113.

VERDIER Benoît, « La communication de la Foi à travers le prisme médiatique d'une série télévisée : Ainsi soient-ils, médiateur d'un croire universel ? ", in Espace Public et Communication de la Foi, éd. Stephan BRATOSIN et Mihaela TUDOR, Montpellier, Editions IARSIC, 2014, p. 229-241.

\section{NOTES}

1. Parmi de nombreux ouvrages sur le sujet, on peut évoquer : Vincent Colonna, L'art des séries télé , Paris, Payot, 2010 ; Jean-Pierre Esquenazi, Les séries télévisées : L'avenir du cinéma ?, Paris, Armand Colin, 2010 ; François Jost, De quoi les séries américaines sont-elles le symptôme ? Paris, CNRS Éditions, 2011 ; mais également la mise en place de collections particulières dans certaines maisons d'éditions notamment celle au PUF dirigée par Jean-Baptiste Jeangène Vilmer et Tristan Garcia.

2. Créée par David Elkaïm, Bruno Nahon, Vincent Poymiro et Rodolphe Tissot, et produite par Zadig Productions et Arte France, les trois saisons de cette série télévisée française Ainsi soient-ils ont été diffusées de 2012 à 2015 sur Arte.

3. Dossier de presse Arte, 2012.

4. Une analyse quantitative, différenciant la citation, de la représentation de l'objet livre, laisse apparaître peu de différences entre les deux états (29 pour 31). Par contre, le manuscrit apparaît 14 fois dans des séquences de rituel pour 17 dans des moments de type profane aussi bien dans le séminaire (bibliothèque, cantine, chambre etc.) qu'à l'extérieur.

5. La durée du montage proposé varie de 33 secondes (1.4) pour la plus courte à 3 minutes 52 secondes (2.3) pour la plus longue.

6. Évangile de Luc, chapitre 16 verset 13.

\section{RÉSUMÉS}

Dans une société marquée par la sécularisation, le concept d' « exculturation » de Danielle Hervieu-Léger rend compte du processus de "dé-liaison », de déconnexion entre la culture collective et la culture de l'église. En outre, dans l'espace public, la religion se trouve régulièrement projetée sur le devant de la scène médiatique, notamment au regard de ses dysfonctionnements (secrets, affaires de pédophilie...). En 2012, une série télévisée française, Ainsi soient-ils, produite et diffusée par Arte, porte le religieux à une heure de large audience. Ponctuellement, les personnages citent de courts passages des écrits consacrés; certaines biographies et études théologiques (de Saint Augustin notamment) sont évoquées. Viennent également dialoguer des textes ou des symboles de type profane.

Cet article interroge cette fiction sérielle à travers le prisme de la littérature dite sacrée. Outre 
les représentations, nous questionnons également les différentes transactions métatextuelles, intertextuelles et interdiscursives qui se font jour tout au long des épisodes. L'objectif de cette contribution est de montrer la capacité d'une série à interroger, à jouer avec les textes sacrés - trop souvent fossilisés par le dogme et le rituel- pour proposer et susciter un questionnement critique social tant sur le croire contemporain que sur les rapports sociaux.

In a society marked by secularization, the Danielle Hervieu-leger's concept of "exculturation" reports the process of "disconnection", a kind of disruption between the plural social collective culture and that one of the church. Besides, in the public sphere, religion is regularly on the media limelight particularly in reference to its dysfunctions (secrets, pedophilia...). In 2012, a french television series called Ainsi soient-ils, produced and broadcasted by Arte, deals with religion at prime time. Characters quote short passages of the sacred texts; some biographies and theological studies (like those of Saint Augustin) are outlined. Profane texts or symbols also come to converse.

This article questions this television series through the lens of the holy writings. Besides representations, we also want to enquire the various metatextual, intertextual and interdiscursive transactions that come forward throughout the episodes. This contribution aims to show the ability of a serial fiction to interrogate, to play with the sacred texts (often fossilized by the dogma and rituals) in order to suggest and provoke an ongoing social critical questioning on about both the contemporary believing and the social relationships.

\section{INDEX}

Mots-clés : religion, texte, sacré, métatextualité, interdiscursivité, intertextualité, réflexivité, Ainsi soient-ils

Keywords : religion, text, sacred, metatextuality, interdiscursivity, intertextuality, reflexivity, Ainsi soient-ils

\section{AUTEUR}

\section{BENOÎT VERDIER}

Benoît Verdier est maître de conférences en Sciences de l'Information et de la Communication à l'Université de Reims Champagne Ardenne. Au sein du CEREP (Centre d'Études et de Recherche sur les Emplois et les Professionnalisations), ses travaux portent actuellement sur le processus de médiatisation des collectifs de travail dans l'information, la fiction et les médias sociaux. Dans ce cadre, il s'intéresse particulièrement à la série télévisée française Ainsi soient-ils qui lui permet d'investiguer ce que les médias font des professionnels du croire et inversement.

Benoît Verdier is a lecturer in Information and Communication Sciences at Reims Champagne Ardenne University. In his research center, CEREP (Centre d'Etudes et de Recherche sur les Emplois et les Professionnalisations), he analyzes the process of mediatization of the occupational group in news, fiction and social media. In this framework, with the striking example of the french TV series Ainsi soient-ils, he investigates how media turn that kind of occupationals (especially in that case, that of the belief) into and vice versa. 